\title{
Subtyping of ST22-MRSA-IV (Barnim epidemic MRSA strain) at a university clinic in Germany from 2002 to 2005
}

Correspondence

B. Ghebremedhin

beniam.ghebremedhin@

medizin.uni-magdeburg.de

Received 10 August 2006

Accepted 20 October 2006

\author{
B. Ghebremedhin, ${ }^{1}$ W. König, ${ }^{1}$ W. Witte, ${ }^{2}$ K. J. Hardy, ${ }^{3}$ P. M. Hawkey ${ }^{3}$ \\ and B. König ${ }^{1}$ \\ ${ }^{1}$ Otto-von-Guericke-University, Institute of Medical Microbiology, Magdeburg, Germany \\ ${ }^{2}$ Robert Koch Institute, Wernigerode, Germany \\ ${ }^{3}$ West Midlands Public Health Laboratory, Health Protection Agency, Birmingham, UK
}

\begin{abstract}
Emergence of the meticillin-resistant Staphylococcus aureus (MRSA) Barnim epidemic strain (ST22-MRSA-IV) was demonstrated recently at University Hospital in Magdeburg, Germany. To aid the study of transmission events, it is important to have an epidemiological typing method with the ability to distinguish among MRSA isolates. The aim of this study was to determine the ability of phenotypic and genotypic methods to type ST22-MRSA-IV strains within a hospital for microevolution events. Forty-two ST22-MRSA-IV strains collected from 2002 to 2005 were analysed using antimicrobial testing, toxin gene analysis, PFGE, spa typing, fluorescent amplified fragment length polymorphism (fAFLP) and determination of staphylococcal interspersed repeat units (SIRUs). Four different antimicrobial patterns were observed. The majority of the isolates ( $n=31$ ) were resistant towards erythromycin, ciprofloxacin and clindamycin, in addition to penicillin and oxacillin. All strains harboured the sec gene and showed a homogeneous profile of toxin genes. One isolate was typed as spa t022, two as spa t474 and the remainder belonged to spa type t032. PFGE yielded eight profiles and SIRU typing resulted in six different patterns. The fAFLP technique subdivided the individual PFGE profiles, but the grouping of isolates differed from that obtained by PFGE or SIRU typing. These results showed a diversity of ST22-MRSA-IV strains within a narrow clinical setting, indicating microevolution of the Barnim MRSA clone. The ability to distinguish among MRSA strains within an endemic setting will lead to a greater understanding of the transmission of MRSA and is necessary to be able to control the spread of various clones.
\end{abstract}

\section{INTRODUCTION}

Meticillin-resistant Staphylococcus aureus (MRSA) emerged in the 1960s and is now commonly seen in hospitals, clinics and, since the mid-1990s, in the community. Epidemic MRSA strains in hospitals are usually clonal in origin. There are only a few pandemic MRSA clones, the so-called Iberian, Brazilian, Hungarian, New York/Japan, paediatric, and epidemic MRSA (EMRSA)-15 and -16 clones, named after the geographical area where they were first identified or

\footnotetext{
Abbreviations: CIP, ciprofloxacin; CLI, clindamycin; EMRSA, epidemic meticillin-resistant Staphylococcus aureus; ERY, erythromycin; fAFLP, fluorescent amplified fragment length polymorphism; FAM, 6-carboxyfluorescein; FUS, fusidic acid; GEN, gentamicin; LIN, linezolid; MLST, multilocus sequence typing; MRSA, meticillin-resistant Staphylococcus aureus; OXA, oxacillin; PEN, penicillin; PVL, Panton-Valentine leukocidin; RCCF, rehabilitation and chronic care facility; RIF, rifampicin; $\mathrm{SCC} m e c$, staphylococcal cassette chromosome mec; SIRU, staphylococcal interspersed repeat unit; SXT, trimethoprim/sulfamethoxazole; TEl, teicoplanin; TET, tetracycline; VAN, vancomycin; VNTR, variablenumber tandem repeat.
}

according to epidemiological characteristics. The strains of these successful lineages are responsible for most hospitalacquired MRSA infections all over the world today (Enright et al., 2002; Robinson \& Enright, 2003). In this regard, the Barnim EMRSA strain (ST22-MRSA-IV) in Germany is ancestral to the UK EMRSA-15 strain (Murchan et al., 2003; Witte et al., 2001).

In a previous study, we analysed 199 MRSA isolates from patients at University Hospital in Magdeburg, Germany, and the rehabilitation and chronic care facility (RCCF) in Flechtingen, Germany (Ghebremedhin et al., 2005) and observed the emergence of the Barnim EMRSA ST22 strain. An understanding of the genetic microevolution and clonal spread of strains is necessary to understand the transmission of MRSA and to control its spread. Phenotypic typing methods are mostly restricted to the resistance pattern of the respective MRSA strains (Berger-Bächi, 2002). At present, there are numerous typing methods available, but there is no consensus regarding which method is the best to apply. 
For epidemiological typing and for studying the dynamics of MRSA evolution in a hospital environment, knowledge of both strain identity and variability within the strain allow us to make judgements on whether direct cross-infection or independent acquisition has taken place. Thus, we need slowly [e.g. multilocus sequence typing (MLST), agr, SCCmec typing] as well as more rapidly [e.g. PFGE, fluorescent amplified fragment length polymorphisms (fAFLP), staphylococcal interspersed repeat units (SIRUs), spa typing] evolving genetic markers to study hospitalacquired MRSA. Methods that index neutral variation, which accumulates slowly in the genome, are better suited to answering global epidemiological questions.

PFGE is a highly discriminatory typing method that is well suited to the investigation of microevolution and recent transmission within a hospital and within a narrow time schedule. However, it has several limitations, including ease of reproducibility and interpretation of band patterns, even when using standardized methods (Murchan et al., 2003). Guidelines and interpretative criteria for the analysis of discrete sets of isolates obtained during outbreaks have been proposed, but these are not appropriate for the analysis of large populations of organisms collected over extended periods. Somehow, PFGE is too discriminatory for studies assessing long-term epidemiology, as factors that may dramatically alter macrorestriction banding profiles (such as insertions, deletions and inversions) may obscure similarity among epidemiologically related isolates (Tenover et al., 1995).

MLST is carried out by sequencing an internal fragment of seven unlinked housekeeping genes. Allelic profiles can be determined, and a clone is defined as a group of isolates having a strictly identical sequence for all seven genes (Enright et al., 2000). MLST examines the slowly evolving genomic core and is a useful tool for defining chromosomal ancestry (Enright et al., 2000). Although MLST is useful for defining evolutionary events, it does not provide a good marker for microevolutionary events within a hospital (Urwin \& Maiden, 2003).

Variable-number tandem repeats (VNTRs) have been used as markers for the strain typing of various bacteria (Francois et al., 2005; Hawkey et al., 2003; Onteniente et al., 2003; van Belkum, 1999). Determination of the number of repeats at each locus produces a digital profile, providing a highly portable typing method allowing comparisons between laboratories. Hardy et al. (2006) previously described the presence of VNTRs in staphylococci. Seven VNTRs, termed SIRUs, distributed around the genome are described, occurring in both unique and multiple sites and varying in length from 48 to $159 \mathrm{bp}$.

fAFLP is a DNA fingerprinting method based on restriction cutting of DNA and stringent PCR amplification of the resulting fragments. fAFLP analysis represents a highly discriminatory and reproducible tool for subtyping genetically homogeneous genomes and identifying outbreak genotypes within bacterial genera (Desai et al., 1998; Hookey et al., 1999; Nair et al., 2000; Thyssen et al., 2000).

The agr locus regulates the production of most staphylococcal exoproteins, including exoenzymes, toxins, surface proteins and other virulence factors, by means of a densitydependent autoinducible signal transduction system driven by a short, post-translationally processed peptide (Ji et al., 1995, 1997).

The mecA gene encoding the alternative penicillin-binding protein (PBP2a) is found on the chromosome in a genomic island designated staphylococcal cassette chromosome mec (SCCmec) (Katayama et al., 2000). At least five major varieties of SCCmec exist and are defined by the specific mec locus present (composed of mecA and its two regulatory genes, mecI and mecRI), as well as the variety of site-specific recombinase genes present (either $c c r A B$ or $c c r C$ ) (Katayama et al., 2000; Ma et al., 2002). SCCmec types I-III are larger elements $(34-67 \mathrm{~kb})$ that tend to contain resistance determinants in addition to mecA and are found more frequently in hospital-acquired MRSA strains (Katayama et al., 2000; Ito et al., 2004). In contrast, our ST22-MRSA strains harboured SCCmec type IV, which can be subtyped again into IVa-d (Okuma et al., 2002; Oliveira \& de Lencastre, 2002), now increasingly recognized as causative agents of community-associated disease in patients that are not affiliated with a hospital or healthcare environment (Zetola et al., 2005).

In the present study, we conducted an analysis of ST22MRSA-IV isolates to evaluate and compare widely used molecular techniques, namely, MLST, SCCmec typing and subtyping, fAFLP and PFGE, for their suitability and usefulness in the typing and fingerprinting of bacterial isolates and in tracking clonal evolution.

\section{METHODS}

Bacterial isolates, isolate identification and antibiotic susceptibility testing. MRSA strains were isolated and identified from various clinical specimens of patients from different departments of University Hospital in Magdeburg, Germany, and the RCCF in Flechtingen, Germany, obtained from August 2002 to April 2005. Identification and antibiotic susceptibility testing [penicillin (PEN), oxacillin (OXA), trimethoprim/sulfamethoxazole (SXT), tetracycline (TET), erythromycin (ERY), clindamycin (CLI), moxifloxacin, gentamicin (GEN), vancomycin (VAN), teicoplanin (TEI), linezolid (LIN), quinopristin/dalfopristin, fosfomycin, fusidic acid (FUS), nitrofurantoin, norfloxacin, ciprofloxacin (CIP), levofloxacin, rifampicin (RIF) and tobramycin] were performed using an automated VITEK 2 system (bioMérieux). The results were interpreted in accordance with current National Committee for Clinical Laboratory Standards guidelines: breakpoints for oxacillin susceptibility used were an MIC of $2 \mathrm{mg} \mathrm{l}^{-1}$ indicating susceptibility and an MIC of $4 \mathrm{mg} \mathrm{l}^{-1}$ indicating resistance, as described previously (Ghebremedhin et al., 2005).

DNA extraction. Strains were grown on brain heart infusion agar or in brain heart infusion broth at $37^{\circ} \mathrm{C}$ overnight. Genomic DNA used as target for all molecular methods was extracted using a Qiagen DNA extraction kit according to the manufacturer's suggestions with 
the modification that $30 \mu \mathrm{g}$ lysostaphin $\mathrm{ml}^{-1}$ and $1 \mathrm{mg}$ lysozyme $\mathrm{ml}^{-1}$ were added at the cell-lysis step. The concentration of DNA was estimated spectrophotometrically (Ghebremedhin et al., 2005).

MLST. MLST was carried out as described by Enright et al. (2000). Analysis of the allelic profile of $S$. aureus isolates was performed using the database of the MLST home web page (http:// www.mlst.net), where seven numbers depicting the allelic profile were assigned that defined the MLST type.

PFGE. All isolates were subjected to PFGE analysis of SmaI-digested chromosomal DNA, which was performed as described by Witte et al. (2004). Fragments of sizes in the range of the ladder (50-1000 kb) were included in the analysis. The criteria for PFGE pattern analysis state that up to three PFGE fragment differences compared with the prototype pattern B1 indicate a close relationship and thus that the isolate is probably a subclone of the prototype (Tenover et al., 1995).

PCR for SCCmec IV subtyping. Analysis of SCCmec type IV was performed as described by Oliveira \& de Lencastre (2002). For differentiation between the SCCmec types IVa and IVb, we used the protocol of Okuma et al. (2002).

agr group-specific multiplex PCR, and Panton-Valentine leukocidin (PVL) gene and toxin gene detection. Extracted genomic DNA was used as a template to amplify specific agr alleles (GenBank accession nos X52543, AF001782, AF001783, AF288215, Z49220, AF346724 and AF346725). For multiplex PCR, one primer set was prepared to amplify the four specific $S$. aureus agr alleles using the primers described by Lina et al. (2003). Details are given in Ghebremedhin et al. (2005). The primers $a g r \mathrm{~B}$ and $\operatorname{agr} \mathrm{C}$ were used to amplify the variable part of the agr operon (1060 bp) with primer annealing conducted at $50{ }^{\circ} \mathrm{C}$ (Strommenger et al., 2004).

The genes sea-see, seg-sej, tsst-1, eta, etb, hlgA, hlgC/B, lukE/D and lukSlukF-PV (lukPV) were detected by PCR, as described previously (Ghebremedhin et al., 2005).

spa gene typing. The procedures described by Harmsen et al. (2003) were used for sequencing and analysis of the spa gene of all of the MRSA isolates.

fAFLP analysis. fAFLP analysis was performed as described by van den Braak et al. (2004). Briefly, $200 \mathrm{ng}$ chromosomal DNA was digested with EcoRI and MseI (both from New England Biolabs) at $37^{\circ} \mathrm{C}$. The DNA fragments were ligated using double-stranded EcoRI and MseI adapters for $2 \mathrm{~h}$ at $37^{\circ} \mathrm{C}$. A pre-selective PCR with adapter-specific primers EcoRI (5'-GACTGCGTACCAGCTT-3') and Mse I (5'-GATGAGTCCTGATCGC-3') was then performed. An aliquot was subjected to a selective PCR in which one of the primers contained additional nucleotides (primer $M s e \mathrm{I}+\mathrm{CC}$ or primer $M s e I+\mathrm{AC})$ at the $3^{\prime}$ end, whilst primer EcoRI was labelled with 6carboxyfluorescein (FAM) at the $5^{\prime}$ end. PCR amplifications were performed with a Perkin-Elmer model 2400 thermocycler using the following protocol: an initial step of $72{ }^{\circ} \mathrm{C}$ for $2 \mathrm{~min}, 20$ cycles of $94{ }^{\circ} \mathrm{C}$ for $1 \mathrm{~s}, 56^{\circ} \mathrm{C}$ for $30 \mathrm{~s}$ and $72^{\circ} \mathrm{C}$ for $2 \mathrm{~min}$, and a final extension step at $72{ }^{\circ} \mathrm{C}$ for $7 \mathrm{~min}$. PCR products were stored at $-20^{\circ} \mathrm{C}$ until analysis of the fAFLP using an automated gel electrophoresis and detection system.

fAFLP fragments were analysed on an ABI Prism 3100 DNA sequencer by adding $2 \mu \mathrm{l}$ of the PCR product to $24 \mu \mathrm{l}$ deionized formamide. To assess the size of the obtained fragments accurately, $1 \mu \mathrm{l} \mathrm{GeneScan-500}$ marker (Applied Biosystems) labelled with the red fluorescent dye 6-carboxy-x-rhodamine was used as internal size standards.

The raw data from the GeneScan were converted to .ab1 format for analysis using BioNumerics software (version 3.0; Applied Maths)
Cluster analysis of the fAFLP patterns was performed with the Dice similarity coefficient using optimization at $1 \%$ and position tolerance and change towards the end of the fingerprint at $1 \%$. Dendrograms were calculated using the unweighted pair-group method with arithmetic mean (UPGMA).

Strains were analysed individually by calculating the number of isolates that clustered together with the number of unique isolates to give a crude measure of the heterogeneity of strain populations.

Tandem repeat locus identification (SIRU typing). SIRU typing of the isolates was conducted according to the method of Hardy et al. (2004) and interpretation of the results was performed according to Hardy et al. $(2004,2006)$.

Statistical analysis. The Hunter (1990) discriminatory index was used to calculate the level of discrimination of each typing method.

\section{RESULTS AND DISCUSSION}

\section{MLST analysis and distribution of ST22-MRSA among different departments at University Hospital}

In a previous study, we detected a dominant MRSA clone, designated Barnim ST22-MRSA, at University Hospital, Magdeburg, and the RCCF, Flechtingen. The allelic profile for the sequence type ST22 was 7-6-1-5-8-8-6 (Ghebremedhin et al., 2005). Meanwhile, the number of ST22-MRSA isolates detected increased to 42 within 24 months. ST22-MRSA isolates were most prevalent in the Department of Dermatology (45.2\%) and at the RCCF in Flechtingen (21.4\%), and $14.3 \%$ of the MRSA isolates were found in distinct ICUs at University Hospital. The remaining strains were distributed among the different departments. The individual numbers of ST22-MRSA isolates for each department over the 24 month study period are given in Table 1 .

MLST has been applied to define the evolutionary relationships of MRSA on a worldwide basis. Although it is useful for analysing evolutionary events, it does not provide a good marker for microevolution events within a hospital (Enright et al., 2000). A combination of MLST with SCCmec typing permits the unambiguous assignment of collections of MRSA isolates to known or new MRSA clones (Enright et al., 2000; Grundmann et al., 2002). Interestingly, in our study, all analysed isolates carried SCCmec subtype IVa (see below), which defined this epidemic MRSA primarily as a regional clone (Shore et al., 2005).

\section{SCCmec typing and subtyping}

To study additional differences among the isolates according to SCCmec IV, subtype analysis was performed by PCR. SCCmec subtypes could be determined for all 42 ST22MRSA strains by PCR and were found to be type IVa.

\section{spa typing}

The spagene of $S$. aureus encodes protein A and was used for typing of the 42 ST22-MRSA-IV isolates using Ridom 
Table 1. Characterization of ST22-MRSA strains by analysis of antimicrobial resistance profile, toxin gene profile, spa type, SIRU profiles and fAFLP fingerprints

\begin{tabular}{|c|c|c|c|c|c|c|c|c|c|c|c|}
\hline Strain & Lab. & No. & Date & Clinic & Antibiotype ${ }^{\star}$ & spa & PFGE & $\begin{array}{c}\text { SIRU } \\
\text { profile }\end{array}$ & fAFLP + AC & fAFLP + CC & $\begin{array}{c}\text { Toxin } \\
\text { profile } \dagger\end{array}$ \\
\hline 23 & VA & 22489 & 29.08 .02 & Endocrinology & Type II & t032 & B1 & $1403 \mathrm{~h}-2$ & I & II & C \\
\hline 1 & BK & 6474 & 30.12 .02 & Cardiothoracic surgery & Type I & t032 & B1 & $1403 \mathrm{~h}-2$ & III & $\mathrm{I}$ & $\mathrm{C}$ \\
\hline 15 & VA & 171 & 03.01 .03 & Dermatology & Type I & $\mathrm{t} 032$ & B1 & $1303 \mathrm{~h}-2$ & III & I & A \\
\hline 20 & VA & 766 & 10.01 .03 & Dermatology & Type I & $\mathrm{t} 032$ & $\mathrm{~B} 1$ & $1403 \mathrm{~h}-2$ & $\mathrm{I}$ & I & A \\
\hline 7 & VA & 2326 & 28.01 .03 & Dermatology & Type I & t032 & $\mathrm{B} 1$ & $1403 \mathrm{~h}-2$ & II & II & A \\
\hline 8 & VA & 2501 & 30.01 .03 & Dermatology & Type I & $\mathrm{t} 032$ & B1 & $1403 \mathrm{~h}-2$ & II & II & A \\
\hline 2 & VA & 2722 & 03.02 .03 & General surgery & Type I & t032 & $\mathrm{B} 1$ & $1403 \mathrm{~h}-2$ & III & II & A \\
\hline 14 & VA & 3415 & 11.02 .03 & Dermatology & Type I & $\mathrm{t} 032$ & $\mathrm{~A} 1$ & $1403 \mathrm{~h}-2$ & III & I & A \\
\hline 37 & VA & 3507 & 12.02 .03 & Flechtingen & Type I & t032 & B1 & $1403 \mathrm{~h}-2$ & III & II & A \\
\hline 33 & VA & 3668 & 13.02 .03 & Anaesthesiology ICU & Type III & $\mathrm{t} 474$ & B2 & $1403 \mathrm{~b}-2$ & II & $\mathrm{I}$ & A \\
\hline 36 & UR & 1611 & 14.02 .03 & Anaesthesiology ICU & Type III & $\mathrm{t} 474$ & B2 & $1403 \mathrm{~b}-2$ & II & III & A \\
\hline 10 & VA & 4055 & 19.02 .03 & Dermatology & Type I & $\mathrm{t} 032$ & A1 & $1303 \mathrm{~h}-2$ & III & IV & A \\
\hline 3 & VA & 4393 & 23.02 .03 & General surgery & Type I & $\mathrm{t} 032$ & B1 & $1403 \mathrm{~h}-2$ & II & I & B \\
\hline 38 & BK & 1061 & 24.02 .03 & Flechtingen & Type I & $\mathrm{t} 032$ & B3 & $1403 \mathrm{~h}-2$ & $\mathrm{I}$ & II & A \\
\hline 19 & VA & 4668 & 26.02 .03 & Dermatology & Type I & t032 & $\mathrm{A} 1$ & $1303 \mathrm{~h}-2$ & III & I & A \\
\hline 11 & UR & 2233 & 04.03 .03 & Dermatology & Type I & $\mathrm{t} 032$ & $\mathrm{~B} 1$ & $1403 \mathrm{~h}-2$ & II & I & A \\
\hline 18 & VA & 5349 & 05.03 .03 & Dermatology & Type I & t032 & Unique $\mathrm{P} 10$ & $1403 \mathrm{~h}-2$ & II & I & A \\
\hline 12 & VA & 6005 & 13.03 .03 & Dermatology & Type I & $\mathrm{t} 032$ & A1 & $1303 \mathrm{~h}-2$ & II & I & A \\
\hline 16 & VA & 6073 & 13.03 .03 & Dermatology & Type I & $\mathrm{t} 032$ & B1 & $1403 \mathrm{~h}-2$ & III & III & A \\
\hline 21 & VA & 6170 & 14.03 .03 & Dermatology & Type I & t032 & B1 & $1403 \mathrm{~h}-2$ & III & III & A \\
\hline 4 & VA & 6194 & 15.03 .03 & General surgery ICU & Type I & t032 & B1 & $1403 \mathrm{~h}-2$ & IV & II & A \\
\hline 17 & VA & 6321 & 17.03 .03 & Dermatology & Type I & t032 & B1 & $1403 \mathrm{~h}-2$ & I & I & A \\
\hline 9 & VA & 6402 & 18.03 .03 & Dermatology & Type I & t032 & B1 & $1403 \mathrm{~h}-2$ & I & II & A \\
\hline 13 & VA & 6861 & 24.03 .03 & Dermatology & Type I & $\mathrm{t} 032$ & B1 & $1403 \mathrm{~h}-2$ & III & II & $\mathrm{C}$ \\
\hline 35 & BK & 2742 & 22.05 .03 & Flechtingen & Type II & t032 & B1 & $1303 \mathrm{~h}-2$ & III & II & A \\
\hline 26 & VA & 13140 & 05.06 .03 & Dermatology & Type I & $\mathrm{t} 032$ & B1 & $1403 \mathrm{~h}-2$ & II & III & A \\
\hline 24 & VA & 13844 & 16.06 .03 & Dermatology & Type III & $\mathrm{t} 032$ & B1 & $1403 \mathrm{~h}-2$ & I & II & $\mathrm{D}$ \\
\hline 25 & VA & 14172 & 19.06 .03 & Dermatology & Type I & $\mathrm{t} 032$ & B1 & $1403 \mathrm{~h}-2$ & I & II & A \\
\hline 27 & VA & 14829 & 27.06 .03 & Otorhinolaryngology & Type I & t032 & B5 & $1603 \mathrm{~h}-2$ & I & II & A \\
\hline 28 & VA & 18312 & 11.08 .03 & Internal medicine ICU & Type I & $\mathrm{t} 032$ & B6 & $1401 \mathrm{~h}-2$ & III & II & A \\
\hline 29 & VA & 19482 & 26.08 .03 & Internal medicine ICU & Type I & t032 & B1 & $1403 \mathrm{~h}-2$ & I & II & $\mathrm{D}$ \\
\hline 31 & VA & 20591 & 08.09 .03 & Neurology & Type III & t032 & B1 & $1403 \mathrm{~h}-2$ & II & I & A \\
\hline 34 & VA & 20537 & 09.09 .03 & Flechtingen & Type II & t032 & B3 & $1403 \mathrm{~h}-2$ & I & II & A \\
\hline 32 & VA & 20728 & 10.09 .03 & Cardiology & Type III & $\mathrm{t} 032$ & B1 & $1403 \mathrm{~h}-2$ & III & III & $\mathrm{C}$ \\
\hline 5 & VA & 24851 & 02.11 .03 & Flechtingen & Type I & $\mathrm{t} 032$ & B1 & $1403 \mathrm{~h}-2$ & II & I & A \\
\hline 30 & VA & 25089 & 05.11 .03 & Anaesthesiology ICU & Type III & t032 & B7 & $1403 \mathrm{~h}-2$ & III & $\mathrm{I}$ & B \\
\hline 6 & BK & 5955 & 14.11 .03 & Flechtingen & Type I & t032 & $\mathrm{B} 1$ & $1403 \mathrm{~h}-2$ & III & III & $\mathrm{B}$ \\
\hline 22 & VA & 27735 & 05.12 .03 & Dermatology & Type I & t032 & $\mathrm{B} 1$ & $1403 \mathrm{~h}-2$ & I & II & $\mathrm{B}$ \\
\hline 39 & $\mathrm{BK}$ & 825 & 24.02 .05 & General surgery & Type I & t032 & $\mathrm{B} 1$ & $1403 \mathrm{~h}-2$ & II & II & A \\
\hline 40 & $\mathrm{BK}$ & 860 & 26.02 .05 & Flechtingen & Type I & $\mathrm{t} 022$ & $\mathrm{~B} 1$ & $1403 g-2$ & I & II & A \\
\hline 41 & VA & 6266 & 31.03 .05 & Flechtingen & Type I & t032 & $\mathrm{B} 1$ & $1403 \mathrm{~h}-2$ & III & II & A \\
\hline 42 & VA & 6928 & 11.04 .05 & Flechtingen & Type I & t032 & $\mathrm{B} 1$ & $1403 \mathrm{~h}-2$ & III & $\mathrm{I}$ & $\mathrm{C}$ \\
\hline
\end{tabular}

*I, PEN-OXA-CIP-CLI-ERY; II, PEN-OXA-CIP-ERY; III, PEN-OXA-CIP.

$\dagger \mathrm{A}, s e c, s e g, s e i, h \lg A, h \lg C / \mathrm{B}, \operatorname{lukD} / \mathrm{E} ; \mathrm{B}$, profile $\mathrm{A}+$ sea; $\mathrm{C}$, profile $\mathrm{A}+\operatorname{lukPV} ; \mathrm{D}, \mathrm{A}+$ sed.

StaphType software. The majority of the ST22 isolates were typed as spa t032 $(n=39 ; 92.9 \%)$ with the repeat succession 26-23-23-13-23-31-29-17-31-29-17-25-17-25-16-28, two isolates were typed as spa 4474 (with the repeat succession 26-23-13-23-31-05-17-25-16-28) and one isolate from the
RCCF in Flechtingen was typed as spa t022 with the repeat succession 26-23-13-23-31-29-17-31-29-17-25-17-25-16-28 (Table 1). The spa type t022 had one deletion in the repeat succession compared with the prototype t032, whereas 474 differed from $\mathrm{t} 032$ by one insertion and seven deletions. spa 
type 4474 was reflected by the PFGE profile B2, whilst spa type t022 belonged to PFGE prototype B1.

spa typing has been shown to be discriminatory enough for outbreak investigation but also to be efficient for global epidemiological studies. The isolates that belonged to spa type 032 revealed a heterogeneous resistance profile, whilst the two isolates with spa type 4744 shared the same resistance phenotype (antibiotype III) (see Table 1). From this result, we concluded that the ST22-MRSA clone may indeed be heterogeneous. Our data support the previous findings that sequence information applied as MLST or spa types is useful for both evolutionary studies and global epidemiological analysis, and to a lesser extent for short-term or local epidemiological analyses (Tang et al., 2000). However, spa typing is more discriminatory compared with MLST data.

\section{PFGE pattern analysis}

PGFE analysis of the 42 ST22-MRSA strains revealed two different restriction patterns, $\mathrm{A}$ and $\mathrm{B}$ (distinguishable by four to five fragment bands), with most of the isolates exhibiting pattern B $(n=37 ; 88.1 \%)$ (Fig. 1). Within pattern A $(n=4)$, we detected no variations. Within the major pattern B, minor variations (up to three bands) allowed their differentiation into the prototype pattern $\mathrm{B} 1$ and the variant patterns B2, B3, B5, B6 and B7 according to O'Neill et al. (2001). One strain showed a pattern variation not described by O'Neill et al. (2001), but fitted PFGE pattern P10 as described by Grady et al. (1999). This 'new' pattern type differed by four bands from pattern B1. The prototype B1 was isolated over the time of the study from different departments at University Hospital, whilst A1 occurred exclusively in the Department of Dermatology between February and March 2003. The profile B2 $(n=2)$ was isolated from two different patients within 2 days in the Anaesthesiology ICU. Strains exhibiting the profile B3 $(n=2)$ were detected at different time points in two specimens that originated from the RCCF in Flechtingen. The additional profiles B5 $(n=1), \mathrm{B} 6(n=1)$ and B7 $(n=1)$ were isolated from different departments at University Hospital only once during the study period.

PFGE indicates variations throughout the chromosome and is the most common genotypic method used in reference and clinical laboratories for typing MRSA. As PFGE analyses genetic markers that undergo rapid evolutionary change, it seems a suitable method to monitor microevolutionary events (Bannermann et al., 1995; Peacock et al., 2002). In our study, we used the high discriminatory power of PFGE for subtyping ST22-MRSA-IV isolates from University Hospital, collected over a time period of 24 months. In total, seven different PFGE patterns were detected. The demonstration of different PFGE profiles within the epidemic Barnim MRSA clone in our study illustrates the role that genetic events such as point mutations, insertions and deletions may play in altering PFGE patterns of closely related isolates. The majority of isolates within the $\mathrm{B}$ cluster differed by one genetic event from the prototype $\mathrm{B} 1$ (B2, B3 and B5). The PFGE pattern B1 is known to be the dominant type strain of ST22-MRSA-IV. For epidemiological typing, knowledge of both strain identity and variability within the strain allows us to make judgements on whether direct cross-infection or independent acquisition has taken place. However, interpretation of PFGE data obtained from a widespread strain such as Barnim MRSA can be problematic with regard to strain variability.

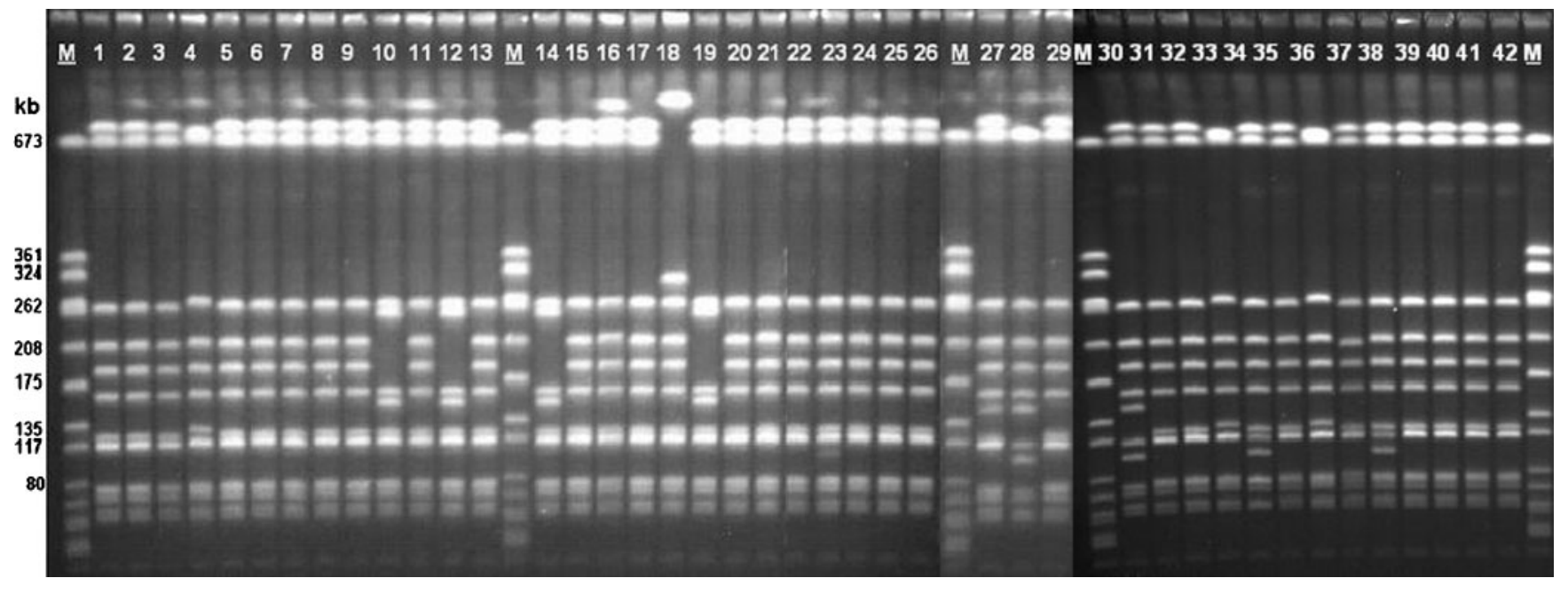

Fig. 1. PFGE of chromosomal DNA Smal restriction of the 42 ST22-MRSA-IV isolates. The spectrum of restriction fragments shown ranges from 10 to $700 \mathrm{~kb}$. Strains were classified into eight pulsotypes (see Table 1); the abundant PFGE pattern represents prototype B1 of ST22-MRSA, whereas A1 is represented by strains $10,12,14$ and 19 . Strain 18 belongs to the unique pattern $\mathrm{P} 10$, strain 30 to B7, and strains 33 and 36 to B2. Strain 27 represents B5 and strain 28 represents B6, whilst B3 is represented by strains 34 and 38 . 
Table 2. Correlation among the various typing methods used for subtyping ST22-MRSA-IV isolates

\begin{tabular}{|c|c|c|c|}
\hline PFGE pattern $(n)$ & Antibiotype $^{\star}(n)$ & spa type $(n)$ & SIRU profile $(n)$ \\
\hline B1 (30) & I (25), II (2), III (3) & t032 (29), t022 (1) & $1403 \mathrm{~h}-2(27), 1303 \mathrm{~h}-2(2), 1403 \mathrm{~g}-2$ (1) \\
\hline B2 (2) & III $(2)$ & $\mathrm{t} 474(2)$ & $1403 b-2(2)$ \\
\hline B5 (1) & I $(1)$ & t032 (1) & $1603 \mathrm{~h}-2(1)$ \\
\hline B6 (1) & I $(1)$ & t032 (1) & $1401 \mathrm{~h}-2(1)$ \\
\hline A1 (4) & I (4) & t032 (4) & $1303 \mathrm{~h}-2(3), 1403 \mathrm{~h}-2(1)$ \\
\hline
\end{tabular}

^I, PEN-OXA-CIP-CLI-ERY; II, PEN-OXA-CIP-ERY; III, PEN-OXA-CIP.

\section{SIRU typing and correlation with PFGE patterns}

PCR was performed on the ST22-MRSA isolates to analyse the discriminatory power of the different loci, as described in Methods. Among the 42 isolates, 6 different SIRU profiles (1303h-2, 1401h-2, 1403b-2, 1403g-2, 1403h-2 and 1603h2 ) were detected (Tables 1 and 2 ). The SIRU profile $1403 \mathrm{~h}-2$ was the predominant type $(n=32 ; 76.2 \%)$. Of these 32 strains, 27 belonged to PFGE profile B1, 2 strains belonged to PFGE profile B3 and 1 strain to PFGE profile A1. The B7 strain and the 'unique' (P10) strain also belonged to SIRU type $1403 \mathrm{~h}-2$. The SIRU type $1603 \mathrm{~h}-2(n=1)$ correlated with PFGE profile B5 $(n=1)$, SIRU type $1403 \mathrm{~b}-2(n=2)$ was associated with both strains of PFGE profile B2 exhibiting the spa type 4744 , and SIRU type $1401 \mathrm{~h}-2(n=1)$ correlated with PFGE profile B6 $(n=1)$. SIRU type $1403 \mathrm{~g}-2(n=1)$ was found to belong to the pulsotype B1 strain but with spa type t022. Surprisingly, SIRU profile $1303 \mathrm{~h}-2(n=5)$ was found within the two major PFGE restriction patterns, B1 $(n=2)$ and A1 $(n=3)$ (Table 2).

According to Hardy et al. (2004), SIRU may be a more appropriate evolutionary marker for studying transmission events compared with PFGE. They showed that the clustering of epidemic strains was the same by SIRU typing and PFGE, but their results indicated that subtyping of EMRSA differed between the two (Hardy et al., 2004, 2006). We have supported and extended their results for the EMRSA-15-derived German epidemic Barnim MRSA clone, ST22-MRSA-IV. The spa gene, encoding protein A, contains a tandem repeat unit VNTR sequence, which was identified as SIRU locus 21. Thus, using SIRU analysis we detected three profiles, 1403h-2, 1403g-2 and 1403b-2, which differed from each other only within SIRU locus 21 . In our study, variation in copy number and DNA sequence within the repeat units of spa was also used as a separate genetic marker (see above). SIRU type 1403g-2 was congruent with spa type t022 and SIRU type 1403b-2 was reflected by spa type 474 . spa type t032 was reflected by five different SIRU profiles (1403g-2, 1403h-2, 1303h-2, 1603h-2 and 1401h-2). In accordance with Malachowa et al. (2005), we concluded that SIRU analysis provides new perspectives on the variability of the $S$. aureus genome and may contribute to a better understanding of the genetic relationships among clones. In this regard, it was interesting to compare the SIRU profiles between EMRSA-15 isolates and isolates belonging to the EMRSA-15-derived Barnim clone. Surprisingly, the collection of EMRSA-15 strains described by Hardy et al. (2004) and the collection of ST22-MRSA-IV strains from our study shared only three SIRU types, 1303h-2, 1403g-2 and 1403h2. Moreover, the most dominant SIRU type, 1203h-2, among the EMRSA-15 strains $(44.3 \%)$ was not present in our study isolates. In contrast, $76.2 \%$ of our study isolates showed the SIRU type $1403 \mathrm{~h}-2$ in comparison with $25.8 \%$ of the EMRSA-15 isolates. Although subtyping of the ST22MRSA strains led to similar results using PFGE and SIRU analysis, SIRU typing was able to distinguish among isolates with the same PFGE profile and vice versa. This has been described previously for bacteria other than S. aureus: Noller et al. (2003) demonstrated the ability of VNTRs to distinguish among isolates with the same PFGE profile during an investigation of an Escherichia coli O157: H7 outbreak. Isolates previously thought to be part of an outbreak according to PFGE were redefined as being sporadic isolates when investigated using VNTRs. Thus, our data add more information on the microevolution of the ancestral EMRSA-15 from the UK.

\section{Cluster analysis of fAFLP data}

fAFLP was used to subtype isolates of the epidemic ST22MRSA. The optimum combination of enzymes and primers for analysis of ST22-MRSA had been predicted by modelling fAFLP using different primer combinations. The primer combinations that were tested initially (EcoRI + A and MseI, $E c o R I+C$ and MseI, EcoRI + AT and MseI, EcoRI and $M s e \mathrm{I}+\mathrm{C}, E c o \mathrm{RI}$ and $M s e \mathrm{I}+\mathrm{AC}$, and EcoRI and MseI + CC) generated fragments ranging in size from 80 to $400 \mathrm{bp}$. We decided to conduct fAFLP using the selective primers MseI + AC or MseI + CC and FAM-labelled EcoRI due to the discriminatory power revealed by these primer combinations. The presence and absence of fragments in each profile were scored. For reasons of reproducibility, the DNA was reextracted and the samples were again subjected to fAFLP. 
The fragment profiles from the different DNA extracts of the same isolate were shown to be nearly identical (data not shown).

According to the fAFLP MseI + AC and FAM-labelled EcoRI fingerprints, analysis of the banding patterns with $1 \%$ optimization and $1 \%$ position tolerance revealed three major clusters and one outlier cluster (Fig. 2). Cluster I $(n=12)$ contained the major relatively homogeneous group of isolates and consisted exclusively of proto-pulsotypes. At $95 \%$ similarity, the cluster could be further subdivided into three subclusters, Ia $(n=10), \mathrm{Ib}(n=1)$ and Ic $(n=1)$. Cluster II $(n=12)$ contained the type strain of the prototype $\mathrm{B} 1(n=8)$, and $\mathrm{A} 1(n=1), \mathrm{P} 10(n=1)$ and $\mathrm{B} 2(n=2)$ strains, which were obtained from patients from different departments and in different isolation periods. The B2 strains that originated from the Anaesthesiology ICU also grouped in this cluster.

The strains of cluster III $(n=17)$, with $66 \%$ similarity, included the majority of the isolates and consisted of prototype $\mathrm{B} 1(n=12), \mathrm{A} 1(n=3), \mathrm{B} 6(n=1)$ and $\mathrm{B} 7(n=1)$.

Cluster IV $(n=1)$ included only one B1 strain. Cluster IV joined the other major clusters at $75.7 \%$ similarity. Thus, A1 strains belonged to two main clusters. In this regard, three isolates of A1 were grouped into cluster III and the remaining one into cluster II.

Using the primer combination MseI + CC and FAM-labelled EcoRI in fAFLP fingerprints, and using $1 \%$ optimization and $1 \%$ position tolerance, three major clusters and one outlier were detected (Fig. 3$)$. Cluster I $(n=15)$ consisted of members of the proto-pulsotype B1 $(n=9), \mathrm{A} 1(n=3), \mathrm{B} 2$ $(n=1), \mathrm{B} 7(n=1)$ and unique $\mathrm{P} 10(n=1)$, whereas the other isolates of B2 grouped into cluster III $(n=6)$, which also included the proto-pulsotype B1 isolates $(n=5)$. Cluster II $(n=20)$ also included the proto-pulsotype B1 isolates $(n=16), \mathrm{B} 3(n=2), \mathrm{B} 5(n=1)$ and $\mathrm{B} 6(n=1)$. Thus, the two isolates that were typed as spa type t474 with SIRU profile 1403b-2 did not cluster in one group. Within the different clusters, further subdivisions could be performed for the isolates of cluster II with a similarity of $88.2 \%$. The outlier isolate joined the other clusters with a similarity of $64.7 \%$.

Grady et al. (1999) described fAFLP analysis as a potent method for subtyping MRSA for the purpose of hospital infection control. In the present study, we asked whether fAFLP was applicable to the subtyping of the major endemic MRSA of MLST type ST22. Indeed, fAFLP was able to discriminate the main PFGE patterns. Furthermore, the additional PFGE patterns also grouped in different clusters of the fAFLP fingerprints. Thus, the discriminatory power of fAFLP, as demonstrated in other studies (Desai et al., 1998; Grady et al., 1999; Harmsen et al., 2003; Tang et al., 2000), was supported in our study and indeed seemed to be superior to PFGE. However, both methods were not concordant in terms of discerning clusters of related isolates.

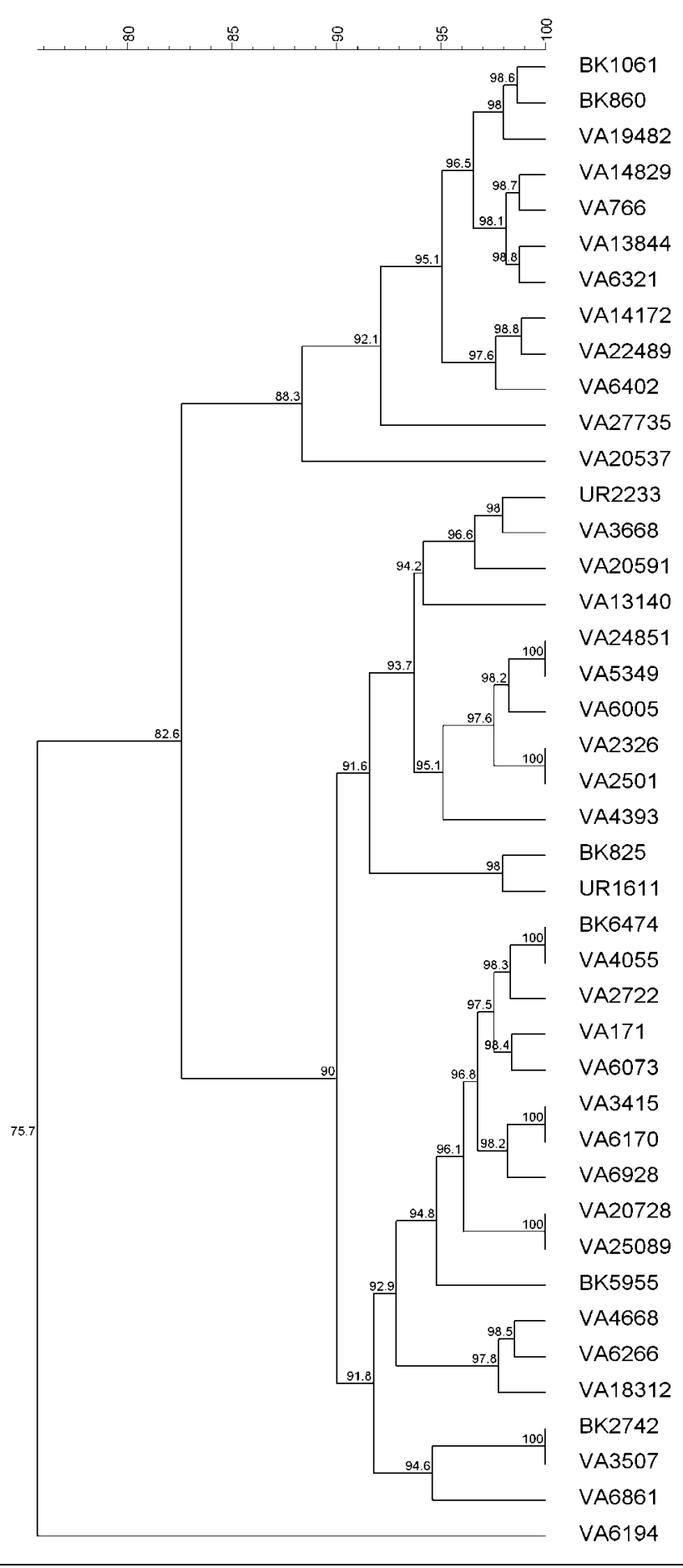

Fig. 2. Dendrogram showing cluster analysis (UPGMA) of the 42 ST22-MRSA-IV isolates based on fingerprints using primers EcoRI and Msel+AC. Results were generated using BioNumerics software with $1 \%$ optimization and $1 \%$ position tolerance.

Thus, fAFLP analysis can be expected to yield insights into the evolution of MRSA as a species. The fAFLP technique samples the whole genome sequence in a predictable and rigorous fashion by monitoring base substitutions 


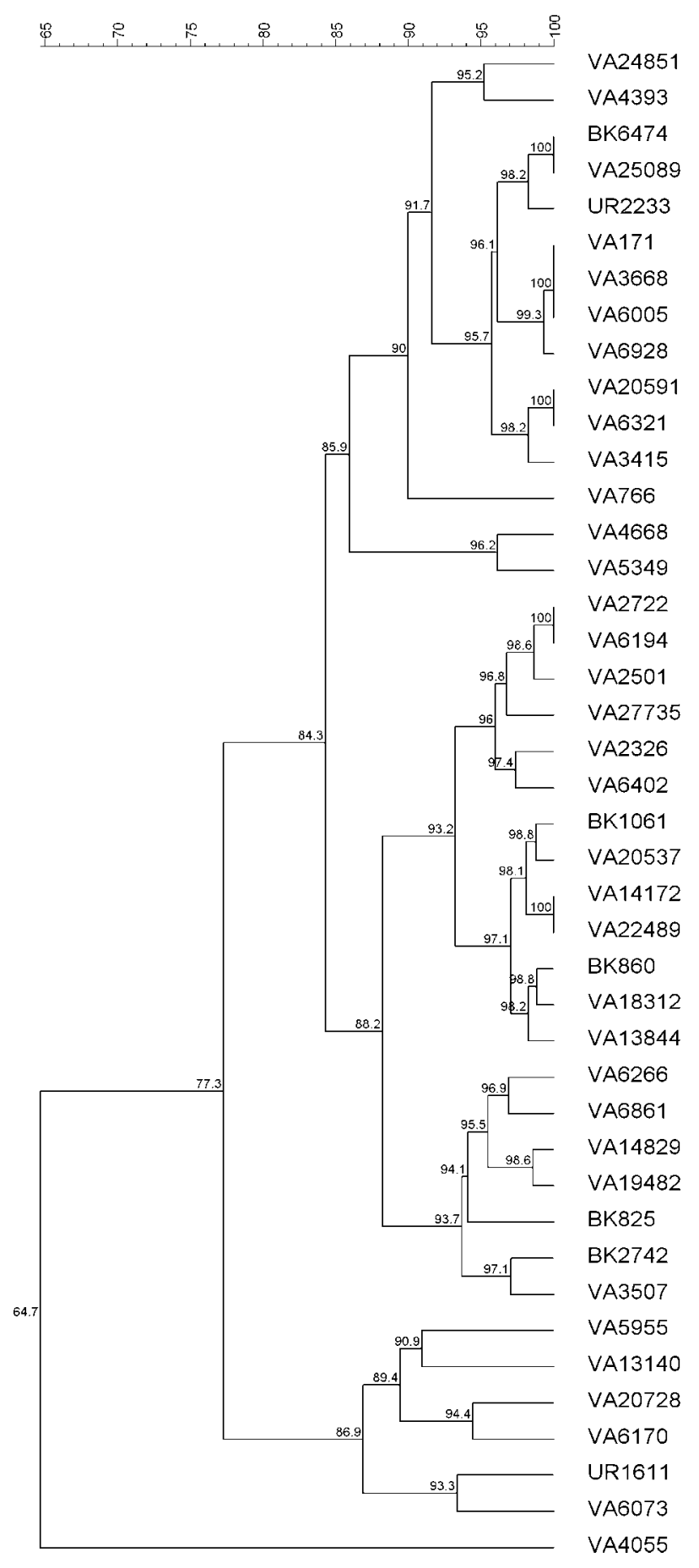

Fig. 3. Dendrogram generated using BioNumerics software showing cluster analysis of the 42 ST22-MRSA-IV isolates based on fingerprints using primers EcoRI and Msel+CC, with $1 \%$ optimization and $1 \%$ position tolerance.

accumulating throughout the genome, rather than being based on the fingerprints of a mobile genetic element. This could provide a valuable measure of microevolutionary change, e.g. within one clone such as ST22-MRSA-IV. The discriminatory power of fAFLP is determined by the choice of restriction enzymes and by the selectivity of the primers used. In case of the two isolates of pulsotype B2, the fAFLP $M s e I+C C$ and EcoRI fingerprints distinguished between these isolates, whereas the fAFLP MseI + AC and EcoRI pattern banding analysis put both isolates of pulsotype $\mathrm{B} 2$ in the same cluster II, which was supported by the SIRU profile pattern and spa typing. In summary, genome-sequencederived fAFLP was broadly congruent with PFGE for typing MRSA. Its resolving power generally appeared to be superior to the other techniques used in this study (Table 3 ). This is particularly the case where strains contain few genetic discriminatory elements and where fAFLP might be capable of revealing chains of transmission not demonstrable by PFGE.

\section{Analysis of toxin genes and the presence of PVL}

We analysed all 42 ST22 strains for the presence of tsst-1, sea-see, seg-sej, the erythrogenic toxin A and B genes eta and $e t b$, the leukocidin genes $h \lg A, h \lg C / B$ and $\operatorname{lukE} / D$, and the PVL gene. The results of the toxin gene analysis are given in Table 1. None of the MRSA strains expressed the eta, etb or tsst-1 gene. Five ST22 isolates carried the PVL gene. All strains possessed the genes for sec, seg, sei, $h \lg A, h \lg C / B$, and $l u k E / D$. In addition to seg and sei, the ST22-MRSA isolates were found to be positive for one or more additional se genes. Thus, in addition to sec, four strains were positive for sea and two for sed (Table 1).

Classical EMRSA-15 is characterized by the production of enterotoxin $\mathrm{C}(\mathrm{sec})$, although enterotoxin C-negative variants of EMRSA-15 have been reported (O'Neill et al., 2001). In our study, all ST22-MRSA isolates carried the gene for enterotoxin $\mathrm{C}$ and showed a homogeneous profile of toxin genes. We observed acquisition of the sea gene in four MRSA strains and acquisition of the PVL gene in five cases. It is known that enterotoxin genes are located on mobile elements. In this regard, the staphylococcal enterotoxin A gene is encoded by phage (Betley \& Mekalanos, 1985). The additional presence of the sea or PVL gene could not be correlated with a specific PFGE pattern profile. All PVL- and sea-positive strains belonged to PFGE type B1 with the exception of one sea-positive strain, which showed PFGE profile B7. The acquisition of toxin genes on mobile elements can go in parallel with extended antibiotic resistance. In our study, the presence of the sea or PVL gene did not change the resistance pattern compared with sea- or PVL-negative strains. As expected, the presence of sea or the PVL gene did not change the SIRU profile or the respective spa type. The fAFLP pattern also was not specifically affected by the acquired toxin genes. In summary, neither of the typing techniques indicated a linkage between genotype and virulence (Hacker et al., 1997).

\section{agr group specificity and agrB-D-C sequencing}

On the basis of the $\operatorname{agrC} 5^{\prime}$ end sequence, all isolates in this study could be grouped into agr group I. Sequencing the 
Table 3. Discriminatory power of the typing methods used in this study

\begin{tabular}{|lcccccc|}
\hline & \multicolumn{6}{c|}{ Method } \\
\cline { 2 - 7 } & Antibiotype & PFGE & spa typing & SIRU & AFLP + AC & AFLP + CC \\
\hline No. of types & 3 & 8 & 3 & 6 & 4 & 4 \\
Discriminatory power & 0.37 & 0.49 & 0.14 & 0.41 & 0.69 & 0.64 \\
\hline
\end{tabular}

variable part (agrB-D-C) of the agr locus revealed no nucleotide changes within this region for any of the ST22MRSA isolates (data not shown).

\section{Antimicrobial resistance phenotypes of the ST22-MRSA isolates}

Initially, we examined the antibiotic resistance phenotypes of all of the ST22-MRSA-IV isolates. All were uniformly sensitive to VAN, TEI and FUS. We found four different resistance phenotypes using a panel of antibiotics (PEN, OXA, SXT, TET, ERY, CLI, GEN, VAN, TEI, LIN, FUS, CIP and RIF). The most common phenotype, PEN-OXA-CIPERY-CLI, named antibiotype I, was present in $78.6 \%$ $(n=33)$ of the ST22 isolates under study. The resistance pattern PEN-OXA-CIP-ERY, named antibiotype II, was detected in $7.1 \%(n=3)$ of the isolates, whereas the resistance pattern PEN-OXA-CIP (antibiotype III) was involved in $14.3 \%(n=6)$ of the isolates. As is apparent from Table 1, the most prominent resistance phenotype, PEN-OXA-CIP-ERY-CLI, was predominant in the Department of Dermatology, University Hospital.

Antibiotype I was observed throughout the study period, whereas antibiotype II was only found in the ST22-MRSA isolates in February 2003. Antibiotype III was characteristic of the ST22-MRSA isolates that were obtained between midJune and November 2003. However, there was no correlation with PFGE pattern, SIRU typing or fAFLP profile, or with toxin profile (Table 2 ).

With regard to the strains under study, we detected three different antibiotypes, PEN-OXA-CIP, PEN-OXA-CIPERY, PEN-OXA-CIP-ERY-CLI. In 1996, the Barnim epidemic MRSA first appeared in German hospitals with the resistance phenotype PEN-OXA-ERY-CIP-CLI. The majority of the studied strains with PFGE profile B1 (80\%) and all with profile $A 1$, the major restriction patterns of ST22-MRSA-IV, showed the original resistance pattern. Thus, $20 \%$ of PFGE prototype B1 $(n=6)$ and some variants of prototype B1, namely B2, B3 and B7, showed a narrowed resistance pattern compared with the main Barnim MRSA strains. Thus, we did not see a strict concordance between antibiotic susceptibility pattern and PFGE pattern. Changes in DNA content due to deleted resistance genes may be too marginal to change the pulsotype (Lipsitch, 2001). This also applies to SIRU typing, spa typing and fAFLP analysis.

\section{Discriminatory power of the typing methods}

Reproducibility was satisfactory with all phenotypic and genotypic typing methods. In accordance with Hunter (1990), a calculator (http://insilico.ehu.es/) was used to determine the discriminatory power of each typing technique used in the study. The most discriminatory methods seemed to be PFGE and fAFLP, followed by SIRU typing and antibiotic resistance phenotyping. The results are given in Table 3. Although there was concordance in general between the different typing methods, it seemed that the more methods used, the more confusion there was in terms of typing. Due to the fact that each method of typing looked at different genetic markers, no single typing technique was really able to decide which was an outbreak and which was an endemic MRSA strain. Nonetheless, to identify microevolutionary events, we propose a combination of two partially contradictory approaches, classification using the most stable markers by MLST and spa and SIRU typing, and subtyping of the bacterial organism by PFGE and fAFLP, in order to yield maximal information about bacterial dissemination.

\section{ACKNOWLEDGEMENTS}

This work was supported by a BMBF-NBL3 grant.

\section{REFERENCES}

Bannermann, T. L., Hancock, G. A., Tenover, F. C. \& Miller, J. M. (1995). Pulsed-field gel electrophoresis as a replacement for bacteriophage typing of Staphylococcus aureus. J Clin Microbiol 33, 551-555.

Berger-Bächi, B. (2002). Resistance mechanisms of Gram-positive bacteria. Int J Med Microbiol 292, 27-35.

Betley, M. J. \& Mekalanos, J. J. (1985). Staphylococcal enterotoxin A is encoded by phage. Science 229, 185-187.

Desai, M., Tanna, A., Wall, R., Efstratiou, A., George, R. \& Stanley, J. (1998). Fluorescent amplified-fragment length polymorphism analysis of an outbreak of group A streptococcal invasive disease. J Clin Microbiol 36, 3133-3137.

Enright, M. C., Day, N. P., Davies, C. E., Peacock, S. J. \& Spratt, B. G. (2000). Multilocus sequence typing for characterization of methicillin-resistant and methicillin-susceptible clones of Staphylococcus aureus. J Clin Microbiol 38, 1008-1015.

Enright, M. C., Robinson, D. A., Randle, G., Feil, E. J., Grundmann, H. \& Spratt, B. G. (2002). The evolutionary history of methicillin-resistant 
Staphylococcus aureus (MRSA). Proc Natl Acad Sci U S A 99, 7687-7692.

Francois, P., Huyghe, A., Charbonnier, Y., Bento, M., Herzig, S., Topolski, I., Fleury, B., Lew, D., Vaudaux, P. \& other authors (2005). Use of an automated multiple-locus, variable-number tandem repeat-based method for rapid and high-throughput genotyping of Staphylococcus aureus isolates. J Clin Microbiol 43, 3346-3355.

Ghebremedhin, B., König, W. \& König, B. (2005). Heterogeneity of methicillin-resistant Staphylococcus aureus strains at a German university hospital during a 1-year period. Eur J Clin Microbiol Infect Dis 24, 388-398.

Grady, R., Desai, M., O’Neill, G., Cookson, B. \& Stanley, J. (1999). Genotyping of epidemic methicillin-resistant Staphylococcus aureus phage type 15 isolates by fluorescent amplified-fragment length polymorphism analysis. J Clin Microbiol 37, 3198-3203.

Grundmann, H., Hori, S., Enright, M. C., Webster, C., Tami, A., Feil, E. J. \& Pitt, T. (2002). Determining the genetic structure of the natural population of Staphylococcus aureus: a comparison of multilocus sequence typing with pulsed-field gel electrophoresis, randomly amplified polymorphic DNA analysis, and phage typing. J Clin Microbiol 40, 4544-4546.

Hacker, J., Blum-Oehler, G., Mühldorfer, I. \& Tschäpe, H. (1997). Pathogenicity islands of virulent bacteria: structure, function and impact on microbial evolution. Mol Microbiol 23, 1089-1097.

Hardy, K. J., Ussery, D. W., Oppenheim, B. A. \& Hawkey, P. M. (2004). Distribution and characterization of staphylococcal interspersed repeat units (SIRUs) and potential use for strain differentiation. Microbiology 150, 4045-4052.

Hardy, K. J., Oppenheim, B. A., Gossain, S., Gao, F. \& Hawkey, P. M. (2006). Use of variations in staphylococcal interspersed repeat units for molecular typing of methicillin-resistant Staphylococcus aureus strains. J Clin Microbiol 44, 271-273.

Harmsen, D., Claus, H., Witte, W., Rothgänger, J., Claus, H., Turnwald, D. \& Vogel, U. (2003). Typing of methicillin-resistant Staphylococcus aureus in a university hospital setting by using novel software for spa repeat determination and database management. J Clin Microbiol 41, 5442-5448.

Hawkey, P. M., Smith, E. G., Evans, J. T., Monk, P., Bryan, G., Mohamed, H. H., Bardhan, M. \& Pugh, R. N. (2003). Mycobacterial interspersed repetitive unit typing of Mycobacterium tuberculosis compared to IS6110-based restriction fragment length polymorphism analysis for investigation of apparently clustered cases of tuberculosis. J Clin Microbiol 41, 3514-3520.

Hookey, J. V., Edwards, V., Patel, S., Richardson, J. F. \& Cookson, B. D. (1999). Use of fluorescent amplified fragment length polymorphism (fAFLP) to characterise methicillin-resistant Staphylococcus aureus. J Microbiol Methods 37, 7-15.

Hunter, P. R. (1990). Reproducibility and indices of discriminatory power of microbial typing methods. J Clin Microbiol 28, 1903-1905.

Ito, T., Ma, X. X., Takeuchi, F., Okuma, K., Yuzawa, H. \& Hiramatsu, K. (2004). Novel type $V$ staphylococcal cassette chromosome mec driven by a novel cassette chromosome recombinase, $c c r C$. Antimicrob Agents Chemother 48, 2637-2651.

Ji, G., Beavis, R. C. \& Novick, R. P. (1995). Cell density control of staphylococcal virulence mediated by an octapeptide pheromone. Proc Natl Acad Sci U S A 92, 12055-12059.

Ji, G., Beavis, R. C. \& Novick, R. P. (1997). Bacterial interference caused by autoinducing peptide variants. Science 276, 2027-2030.

Katayama, Y., Ito, T. \& Hiramatsu, K. (2000). A new class of genetic element, staphylococcus cassette chromosome mec, encodes methicillin resistance in Staphylococcus aureus. Antimicrob Agents Chemother 44, 1549-1555.
Lina, G., Boutite, F., Tristan, A., Bes, M., Etienne, J. \& Vandenesch, F. (2003). Bacterial competition for human nasal cavity colonization: role of staphylococcal agr alleles. Appl Environ Microbiol 69, 18-23. Lipsitch, M. (2001). The rise and fall of antimicrobial resistance. Trends Microbiol 9, 438-444.

Ma, X. X., Ito, T., Tiensasitorn, C., Jamklang, M., Chongtrakool, P., Boyle-Vavra, S., Daum, R. S. \& Hiramatsu, K. (2002). Novel type of staphylococcal cassette chromosome mec identified in communityacquired methicillin-resistant Staphylococcus aureus strains. Antimicrob Agents Chemother 46, 1147-1152.

Malachowa, N., Sabat, A., Gniadkowski, M., Krzyszton-Russjan, J., Empel, J., Miedzobrodzki, J., Kosowska-Shick, K., Appelbaum, P. C. \& Hryniewicz, W. (2005). Comparison of multiple-locus variablenumber tandem-repeat analysis with pulsed-field gel electrophoresis, spa typing, and multilocus sequence typing for clonal characterization of Staphylococcus aureus isolates. J Clin Microbiol 43, 3095-3100.

Murchan, S., Kaufmann, M. E., Deplano, A., de Ryck, R., Struelens, M., Elsberg Zinn, C., Fussing, V., Salmenlinna, S., Vuopio-Varkila, J. \& other authors (2003). Harmonization of pulsed-field gel electrophoresis protocols for epidemiological typing of strains of methicillin-resistant Staphylococcus aureus: a single approach developed by consensus in 10 European laboratories and its application for tracing the spread of related strains. J Clin Microbiol 41, 1574-1585.

Nair, S., Schreiber, E., Thong, K. L., Pang, T. \& Altwegg, M. (2000). Genotypic characterization of Salmonella typhi by amplified fragment length polymorphism fingerprinting provides increased discrimination as compared to pulsed-field gel electrophoresis and ribotyping. J Microbiol Methods 41, 35-43.

Noller, A. C., McEllistrem, M. C., Pacheco, A. G., Boxrud, D. J. \& Harrison, L. H. (2003). Multilocus variable-number tandem repeat analysis distinguishes outbreak and sporadic Escherichia coli O157: H7 isolates. J Clin Microbiol 41, 5389-5397.

Okuma, K., Iwakawa, K., Turnidge, J. D., Grubb, W. B., Bell, J. M., O'Brien, F. G., Coombs, G. W., Pearman, J. W., Tenover, F. C. \& other authors (2002). Dissemination of new methicillin-resistant Staphylococcus aureus clones in the community. J Clin Microbiol 40, 4289-4294.

Oliveira, D. C. \& de Lencastre, H. (2002). Multiplex PCR strategy for rapid identification of structural types and variants of the mec element in methicillin-resistant Staphylococcus aureus. Antimicrob Agents Chemother 46, 2155-2161.

O'Neill, G. L., Murchan, S., Gil-Setas, A. \& Aucken, H. M. (2001). Identification and characterization of phage variants of a strain of epidemic methicillin-resistant Staphylococcus aureus (EMRSA-15). J Clin Microbiol 39, 1540-1548.

Onteniente, L., Brisse, S., Tassios, P. T. \& Vergnaud, G. (2003). Evaluation of the polymorphisms associated with tandem repeats for Pseudomonas aeruginosa strain typing. J Clin Microbiol 41, 4991-4997.

Peacock, S. J., de Silva, G. D., Justice, A., Cowland, A., Moore, C. E., Winearls, C. G. \& Day, N. P. (2002). Comparison of multilocus sequence typing and pulsed-field gel electrophoresis as tools for typing Staphylococcus aureus isolates in a microepidemiological setting. J Clin Microbiol 40, 3764-3770.

Robinson, D. A. \& Enright, M. C. (2003). Evolutionary models of the emergence of methicillin-resistant Staphylococcus aureus. Antimicrob Agents Chemother 47, 3926-3934.

Shore, A., Rossney, A. S., Keane, C. T., Enright, M. C. \& Coleman, D. C. (2005). Seven novel variants of the staphylococcal chromosomal cassette mec in methicillin-resistant Staphylococcus aureus isolates from Ireland. Antimicrob Agents Chemother 49, 2070-2083.

Strommenger, B., Cuny, C., Werner, G. \& Witte, W. (2004). Obvious lack of association between dynamics of epidemic methicillin-resistant Staphylococcus aureus in central Europe and agr specificity groups. Eur J Clin Microbiol Infect Dis 23, 15-19. 
Tang, Y.-W., Waddington, M. G., Smith, D. H., Manahan, J. M., Kohner, P. C., Highsmith, L. M., Li, H., Cockerill, F. R., III, Thompson, R. L. \& other authors (2000). Comparison of protein A gene sequencing with pulsed-field gel electrophoresis and epidemiologic data for molecular typing of methicillin-resistant Staphylococcus aureus. J Clin Microbiol 38, 1347-1351.

Tenover, F. C., Arbeit, R. D., Goering, R. V., Mickelsen, P. A., Murray, B. E., Persing, D. H. \& Swaminathan, B. (1995). Interpreting chromosomal DNA restriction patterns produced by pulsed-field gel electrophoresis: criteria for bacterial strain typing. J Clin Microbiol 33, 2233-2239.

Thyssen, A., van Eygen, S., Hauben, L., Goris, J., Swings, J. \& Ollevier, F. (2000). Application of AFLP for taxonomic and epidemiological studies of Photobacterium damselae subsp. piscicida. Int J Syst Evol Microbiol 50, 1013-1019.

Urwin, R. \& Maiden, M. C. J. (2003). Multi-locus sequence typing: a tool for global epidemiology. Trends Microbiol 11, 479-487. van Belkum, A. (1999). The role of short sequence repeats in epidemiologic typing. Curr Opin Microbiol 2, 306-311.

van den Braak, N., Simons, G., Gorkink, R., Reijans, M., Eadie, K., Kremers, K., van Soolingen, D., Savelkoul, P., Verbrugh, H. \& van Belkum, A. (2004). A new high-throughput AFLP approach for identification of new genetic polymorphism in the genome of the clonal microorganism Mycobacterium tuberculosis. J Microbiol Methods 56, 49-62.

Witte, W., Enright, M., Schmitz, F. J., Cuny, C., Braulke, C. \& Heuck, D. (2001). Characteristics of a new epidemic MRSA in Germany ancestral to United Kingdom EMRSA 15. Int J Med Microbiol 290, 677-682.

Witte, W., Cuny, C., Strommenger, B., Braulke, C. \& Heuck, D. (2004). Emergence of a new community acquired MRSA strain in Germany. Euro Surveill 9, 16-18.

Zetola, N., Francis, J. S., Nuermberger, E. L. \& Bishai, W. R. (2005). Community-acquired meticillin-resistant Staphylococcus aureus: an emerging threat. Lancet Infect Dis 5, 275-286. 